\title{
On the conditions of carbon nanotube growth in the arc discharge
}

\author{
M Keidar and A M Waas \\ Department of Aerospace Engineering, University of Michigan, Ann Arbor, MI 48109, USA
}

Received 29 March 2004, in final form 4 August 2004

Published 1 October 2004

Online at stacks.iop.org/Nano/15/1571

doi:10.1088/0957-4484/15/11/034

\begin{abstract}
The conditions for single wall carbon nanotube formation in the arc discharge method of nanotube production are described. Carbon nanotube seed formation and charging in the interelectrode gap are found to be very important effects that may alter carbon nanotube formation on the cathode surface. The model predicts that the long carbon nanotubes formed in the relatively dense plasma region can be deposited on the cathode surface. The nanotubes in the cathode deposit are primarily oriented in the cathode surface plane and not along the electric field. This prediction is qualitatively confirmed by an SEM analysis of the cathode deposit.
\end{abstract}

(Some figures in this article are in colour only in the electronic version)

\section{Introduction}

Carbon nanotubes (CNTs) are tubular carbon-based structures, produced from graphitic carbon. Since the discovery of CNTs synthesized by arc discharge [1], interest in CNTs has been stimulated by their unique mechanical, thermal and electrical properties and potential applications that exploit these properties. CNTs are believed to be promising candidates for use in field-emission displays [2, 3], nanoelectronics [4], hydrogen storage [5], chemical gas sensors [6] etc.

Among several methods for preparing CNTs, arc discharge is the most practical one for scientific purposes. The arc discharge method yields highly graphitized tubes, because the manufacturing process occurs at a very high temperature. CNT growth conditions depend on the background pressure in helium and argon atmosphere. In the He-Ar mixture it was found that the argon mole fraction affects the CNT diameter [7], while the CNT diameter was found to be fairly independent of pressure in the pure helium environment [8]. In addition to the CNT diameter, two other parameters are important for CNT applications, namely chirality and aspect ratio [9]. The chiral angle determines whether the CNT has metallic or semiconductor electrical conductivity [10].

Several models were proposed to explain the CNT formation in an arc discharge. The CNT formation process on a nanoscopic level is the result of collisions between clusters, ions and neutrals leading to particle attachment. There is some controversy in the literature about the process leading to CNT formation in an arc discharge. Gamaly and
Ebbesen [11] presented probably the first detailed analysis of the arc discharge and the model of CNT formation. They proposed that the bimodal carbon velocity distribution (ions with drift velocity and isotropic neutrals) determines the nanotube creation process near the cathode. According to this model, most nanotubes are produced in the central part of a rodlike deposit on a flat cathode surface. Therefore they claimed that isotropic distribution leads to fullerene formation while directed flux results in nanotubes. Iijima et al [12] proposed an open-ended growth model. In this model, carbon atoms and small carbon clusters add on to the reactive dangling bonds at the edges of the open-ended nanotubes. Other researchers argue that CNTs are elongated by electrostatic forces along the electric field near the cathode [13, 14]. However, high-resolution transmission electron microscopy analysis does not support this hypothesis. Therefore a twostep growth model has been proposed [15]. In this model, first, different carbon structures are formed. Then during the cooling process, the graphitization occurs from the surface towards the interior of the assemblies.

There is additional controversy regarding the region of the nanotube formation. It was mentioned above that CNTs are formed in abundance (about 66\%) in a central part of a rodlike cathode deposit [11]. Significant nanotube amounts in the deposit were also mentioned by Farhat et al [7]. They claimed that the cathode deposit contains the highest density of CNTs. On the other hand, Ando et al [16] claimed that virtually no single wall nanotubes can be found in the cathode deposit. In fact they proposed a cathode design with an oblique angle of 
about $30^{\circ}$ from the anode to reduce the cathode deposit and to increase nanotube mass production.

The main objective of this paper is to formulate the conditions for CNT formation in different regions of the arc discharge chamber. We consider typical conditions in the anodic arc discharge leading to the formation of single wall nanotubes (SWNTs).

\section{Physical model and mathematical description}

Typically plasma density has its highest value along the arc discharge centreline due to plasma outflow in the radial direction [17]. In addition to that, ion acceleration towards the cathode leads to lower plasma density near the cathode surface. Therefore, the probability of collisions and thus nanotube seed formation should be higher in the interelectrode gap and near the anode. In this paper we consider single wall nanotube (SWNT) formation which is typical for an anodic arc discharge [8]. It should be noted that in this case the catalytic particles are involved in the nucleation process [8]. However, catalytic particle interaction with carbon nanotubes is not considered in this paper since the main objective of this study is the condition of CNT deposition on the cathode which does not depend on this interaction. Initial seed particles can be formed in the interelectrode plasma as a result of collisions between clusters, ions and neutrals. Previously it was concluded that the local thermodynamic equilibrium (LTE) is established during the anodic arc duration [8, 17, 18]. Therefore the typical range of plasma parameters (plasma density, electron temperature) used for evaluation of the CNT interaction with the discharge plasma is taken from previous studies in which LTE was considered. Numerous experimental and theoretical studies revealed that the plasma density in the interelectrode gap of the anodic arc is about $10^{15}-10^{16} \mathrm{~cm}^{-3}$, and the electron temperature is about $0.5-1 \mathrm{eV}[8,17,18]$. However, an additional effect should be considered, namely electrical charging of the seed particles [19]. The interaction of the seed particle with the plasma leads to the development of a negative charge on the seed particle due to higher electron mobility. The kinetics of the seed particle charging is determined by the ion and electron flux, which is dependent on the potential distribution in the electric sheath surrounding the seed particle.

From the interelectrode region, the carbon and metal particles as well as seed particles flow to the cathode, cathode deposit and discharge chamber wall as schematically shown in figure 1. First, let as consider the conditions of the seed particle condensation on the cathode and the cathode deposit surface. Charged particles interact with an electric field in the cathode sheath. In addition, charged seed particles generate an electric field and induce an electric dipole moment in surrounding particles. This interaction between seed particles may lead to aligning with the electric field and the production of the ordered linear structures. CNT seeds are generally repelled in the cathode sheath, since CNT seeds are charged negatively in the interelectrode plasma, and in the cathode sheath the potential drop is expected to be negative. However, depending on the momentum that is transferred from the ion flux and the CNT initial velocity at the sheath edge, some seed particles can be deposited on the cathode surface. Thus, an analysis of CNT seed (or just CNT) transport near the cathode region should

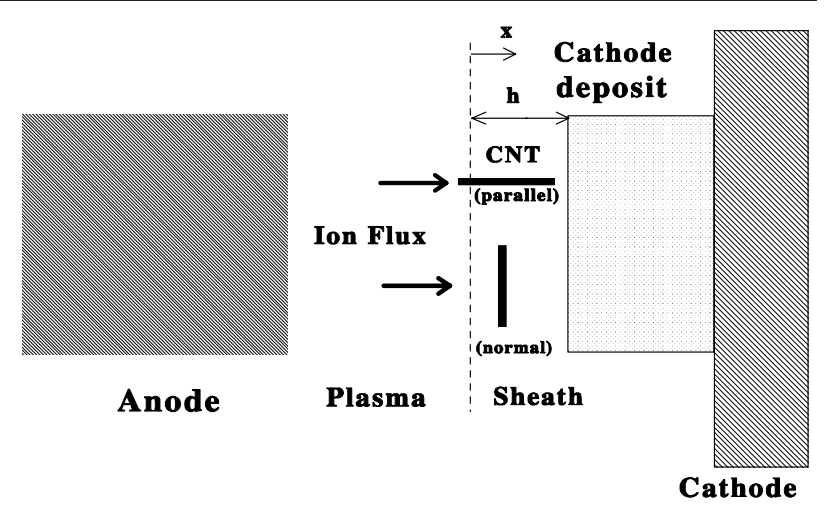

Figure 1. Schematic drawing of the arc discharge and CNT formation and transport in the sheath.

help to establish the conditions for seed particle deposition on the cathode surface and therefore the preferred region for CNT growth in the arc discharge as well as the CNT properties (aspect ratio).

We start with a brief description of the sheath near the cathode in which the CNT interacts with an electric field [20]. In the absence of ionization in the sheath, the ions are described by the following system of equations [20]:

$$
\begin{gathered}
\frac{\mathrm{d}\left(n_{\mathrm{i}} V_{\mathrm{i}}\right)}{\mathrm{d} x}=0 \\
m_{\mathrm{i}} V_{\mathrm{i}} \frac{\mathrm{d} V_{\mathrm{i}}}{\mathrm{d} x}=-Z_{\mathrm{i}} e \frac{\mathrm{d} \varphi}{\mathrm{d} x}
\end{gathered}
$$

where $x$ is the coordinate having its origin at the plasma-sheath interface and directed normal to the wall (see figure 1), $n_{\mathrm{i}}, V_{\mathrm{i}}$, and $Z_{\mathrm{i}}$ are the ion density, velocity and mean charge, and $\varphi$ is the potential. The usual sheath existence criterion is used, i.e. the ion velocity at the sheath edge is equal to the Bohm velocity [21]. The electron density in the sheath obeys the Boltzmann relation:

$$
n_{\mathrm{e}}=n_{0} \exp (e \varphi / k T)
$$

where $n_{0}$ is the electron density at the plasma-sheath interface and the plasma is assumed to be quasi-neutral at the sheath edge. The potential distribution in the sheath can be calculated from the Poisson equation:

$$
\frac{\mathrm{d}^{2} \varphi}{\mathrm{d} x^{2}}=\frac{e}{\varepsilon_{0}}\left(n_{\mathrm{e}}-Z_{\mathrm{i}} n_{\mathrm{i}}\right) .
$$

The boundary conditions for the Poisson equation are: $\varphi / x=0=(\mathrm{d} \varphi / \mathrm{d} x) / x=0=0$ and $\varphi(x=h)=U_{\mathrm{c}}$, where $h$ is the sheath thickness (see figure 1) and $U_{\mathrm{c}}$ is the cathode sheath potential drop. Additionally, the equation for CNT trajectory in the sheath reads as follows [20,22]:

$$
M_{\mathrm{C}} \frac{\mathrm{d}^{2} x}{\mathrm{~d} t^{2}}=\rho \sigma\left(V_{\mathrm{i}}-V_{\mathrm{C}}\right)^{2}-Q_{\mathrm{C}} \frac{\mathrm{d} \varphi}{\mathrm{d} x}
$$

where $M_{\mathrm{C}}, V_{\mathrm{C}}$, and $Q_{\mathrm{C}}$ are the CNT mass, velocity and charge, $\rho$ is the mass plasma density, and $\sigma$ is the momentum transfer cross section. The first term on the left-hand side of this equation is the drag force for the case of free molecule flow (Knudsen number, $K_{n} \gg 1$ ) and for the plasma with a large 
On the conditions of carbon nanotube growth in the arc discharge

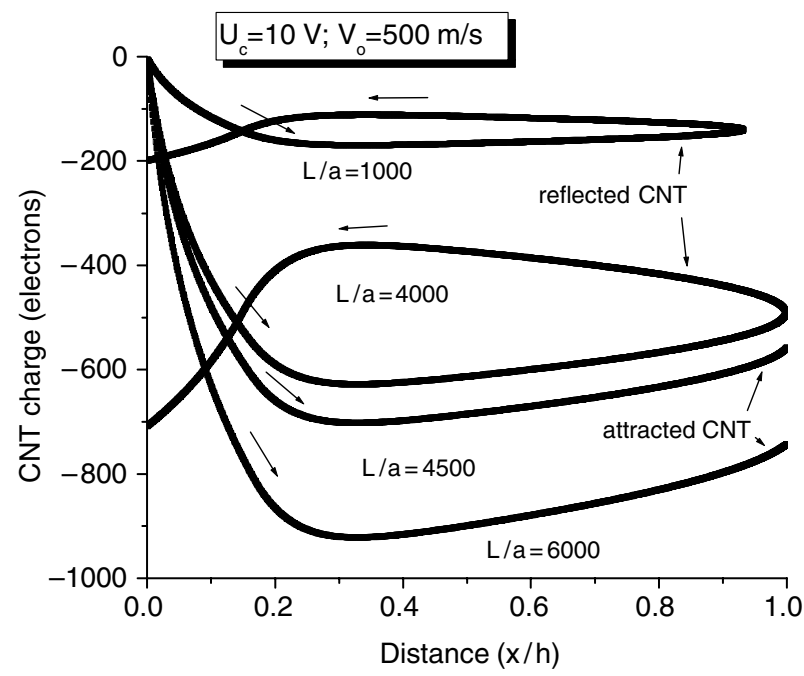

Figure 2. Spatial distribution of CNT charge in the near cathode sheath with aspect ratio as a parameter. The arrow indicates the direction of the CNT charge evolution. $x$ is the distance from the sheath edge towards the cathode.

directed velocity. In order to calculate the electron and ion fluxes to the CNT, the capacitance of the cylindrical particle must be calculated. Following [23], the electric capacitance of the cylindrical particle is estimated as: $C=4 \pi \varepsilon_{0} \frac{L}{\ln (2 L / a)}$, where $L$ is the CNT length and $a$ is the CNT radius. The capacitance does not depend on the inner radius of the CNT since it is calculated between the CNT and the surrounding plasma. Taking into account that the CNT is a conductor (having either metallic or semiconductor properties), the CNT charge is equal to $Q_{\mathrm{C}}=C \varphi_{\mathrm{C}}$, where $\varphi_{\mathrm{C}}$ is the CNT potential with respect to the local potential in the sheath. The CNT charge evolution is calculated as $\mathrm{d} Q / \mathrm{d} t=I_{\mathrm{I}}-I_{\mathrm{e}}$, where $I_{\mathrm{i}}$ is the total ion current and $I_{\mathrm{e}}$ is the total electron current collected by the CNT in the sheath $[20,22]$.

Equation (5) for the CNT trajectory should be supplemented by the boundary conditions, i.e. CNT velocity at the sheath edge. The velocity that the CNT can gain in the interelectrode gap due to interaction with flowing plasma can be readily estimated to be dependent on the CNT orientation with respect to the plasma flow, as shown in figure 1 . The electric field in the interelectrode gap is small and therefore can be neglected. Using the equation of motion (the same as in the sheath, i.e. equation (5)), one can estimate the upper limit for the CNT velocity in the case of the normal CNT orientation (see figure 1) with respect to the plasma flow:

$$
V_{0} \approx \frac{\sigma \rho V_{\mathrm{i}}^{2} t}{M_{\mathrm{C}}} \approx \frac{n b^{2} V_{\mathrm{i}}^{2} t}{\pi}
$$

where $n$ is the plasma density, $V_{\mathrm{i}}$ is the ion velocity, $t$ is the time of CNT-plasma interaction in the interelectrode gap, and $b$ is the $\mathrm{C}-\mathrm{C}$ spacing in CNT.

In the other limiting case, i.e. CNT orientation along the flow or parallel orientation (see figure 1), the CNT has smaller cross section for momentum transfer and therefore the CNT velocity has the following upper limit:

$$
V_{0} \approx \frac{a n b^{2} V_{\mathrm{i}}^{2} t}{L}
$$

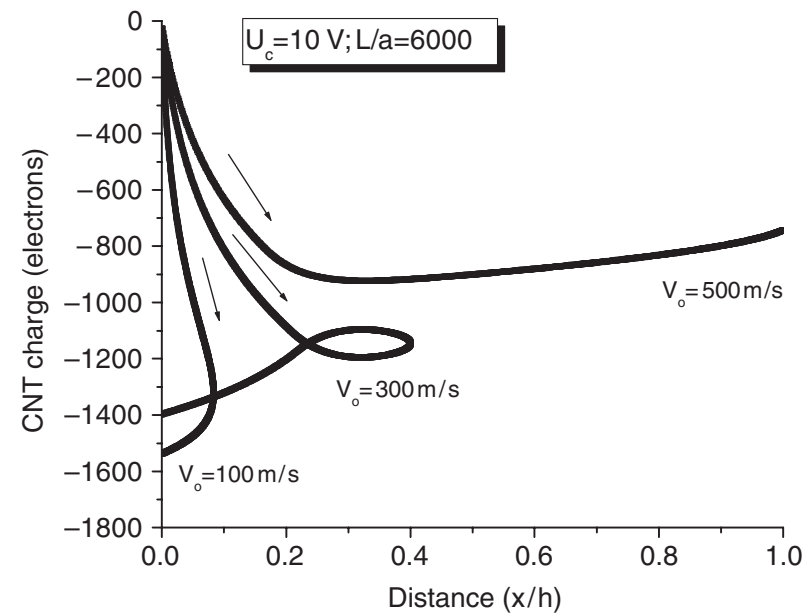

Figure 3. Spatial distribution of CNT charge in the near cathode sheath with initial CNT velocity as a parameter. The arrow indicates the direction of the CNT charge evolution. $x$ is the distance from the sheath edge towards the cathode.

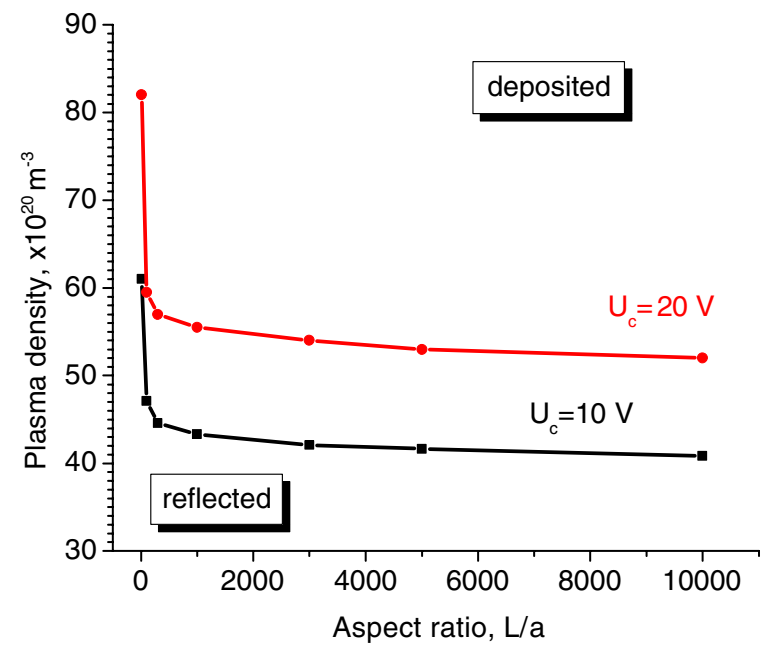

Figure 4. Parameter range for reflected and deposited CNTs.

Thus, for a large aspect ratio CNT $(L / a)$ one can see that the difference between these two cases may be very large. It will be shown below that only CNTs having orientation normal to the flow can reach the cathode surface. Assuming that the CNT has C-C spacing $b=1.4 \AA$ [24] and ion velocity towards the cathode is close to the thermal, $V_{\mathrm{i}}=\sim 3.5 \times 10^{3} \mathrm{~m} \mathrm{~s}^{-1}$, one can estimate that the CNT velocity may be as high as $1000 \mathrm{~m} \mathrm{~s}^{-1}$. Therefore the maximal time for CNT interaction in the interelectrode gap is about $10^{-6} \mathrm{~s}$ for the case of an interelectrode gap of about $1 \mathrm{~mm}$.

\section{Results}

The calculated CNT charge evolution in the sheath is shown in figure 2, where the CNT aspect ratio (length-to-radius ratio) is used as a parameter. Initially, at the plasma-sheath interface, the CNT has a negative charge, corresponding to steady state charging in the quasi-neutral plasma in the interelectrode gap. One can see that the CNT can transit through the sheath or can be reflected due to electrostatic interactions dependent on 


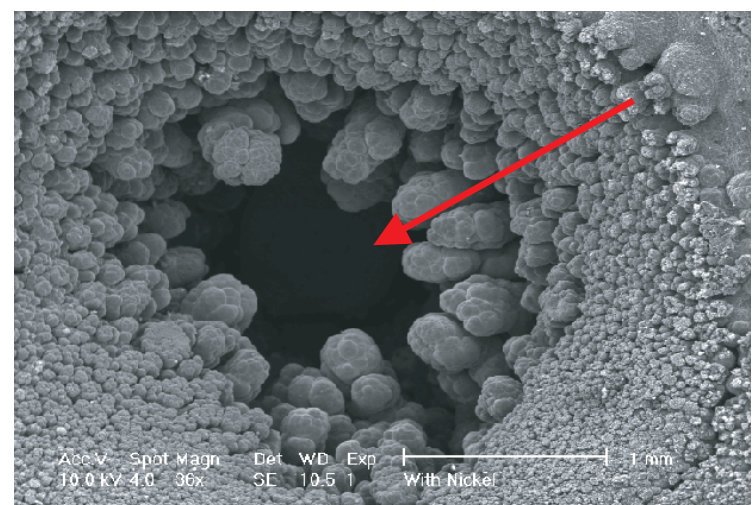

(a)

Figure 5. View of the cathode deposit from the top (SEM).

the aspect ratio. Only long CNTs (large $L / a$ ) are attracted and can finally reach the cathode surface according to these predictions. In addition, an initial CNT velocity at the sheath entrance affects the CNT motion in the sheath, as shown in figure 3. One can see that CNTs having high velocity can reach the cathode, while CNTs having relatively small velocity at the sheath edge are reflected due to interaction with an electric field in the sheath. Thus, according to our calculations, the CNT dynamics in the sheath depends on the CNT velocity at the sheath edge, CNT aspect ratio and the potential drop across the cathode sheath (cathode voltage). On the other hand, the CNT velocity at the sheath edge depends upon the plasma density in the interelectrode gap for a given discharge geometry (according to equations (6), (7)). In addition, only CNTs having a normal orientation with respect to the ion flux can develop the necessary velocity to overcome the repulsive interaction with the electric field in the sheath. Based on the CNT motion analysis in the sheath, one can find CNT and arc discharge parameters $(L / a$ and $n)$ that allow CNT deposition on the cathode. These results are summarized in figure 4, where the cathode voltage drop is used as a parameter. The parameter envelope above the curve provides the necessary conditions for CNT deposition at the cathode surface. It can be seen that CNTs having large aspect ratio can overcome the potential barrier and transit through the cathode sheath and therefore can be found in the cathode deposit.

It was shown that only normally oriented CNTs can be found in the cathode deposit, since they can develop high velocity in the plasma and overcome the cathode potential drop. Therefore, one can conclude that CNT alignment along the electric field is not likely to happen in the cathode deposit. Studying scanning electron microscopy (SEM) images of the cathode deposit can help to verify this prediction. A typical SEM image of the cathode deposit is shown in figure 5. It can be seen that the morphology of the top section of the cathode deposit is rather uniform without any columnar structure in the direction of the electric field. Typically the aspect ratio of CNT in the cathode deposit is about 1000 and larger, which is in agreement with our model prediction. Thus, this observation, in principle, confirms our model prediction. Finally, we found that the large plasma density during the arc discharge provides high CNT velocity at the sheath edge due to momentum interaction. It is known that the plasma

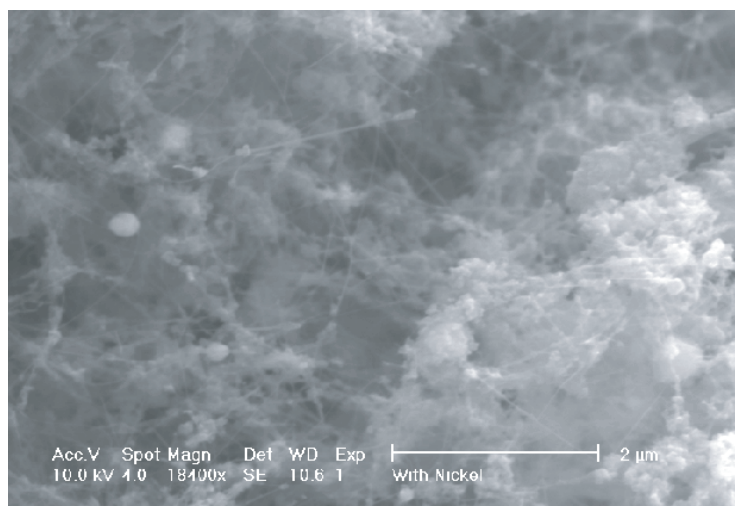

(b)

(a) General view; (b) magnified view of the cathode deposit.

density increases with the discharge current in the anodic arc discharge [18]. This implies that the possibility of CNT growth in the cathode deposit should increase with increasing arc current. This model prediction is in agreement with our experimental observations.

\section{Summary}

In this paper the conditions for carbon nanotube formation in the arc discharge have been clarified. It was shown that CNT seed formation and charging in the interelectrode gap critically affect the conditions of nanotube deposition on the cathode surface and therefore nanotube growth in the cathode deposit. Long carbon nanotubes formed in the relatively dense plasma region can be deposited on the cathode surface. CNTs having smaller aspect ratio can be dragged by the plasma jet and consequently deposit on the chamber walls. Therefore, using an external magnetic field to stabilize the arc discharge and to increase the plasma density would be beneficial, since the CNT density in the cathode deposit can be increased. The present model also predicts that deposited CNTs would be primarily oriented in the cathode surface plane and not along the electric field. This prediction was confirmed by SEM analysis of the cathode deposit.

\section{Acknowledgments}

The authors thank undergraduate research students Daniel Tan and Joe Lohrum for help with carbon nanotube preparation and SEM analyses and the University of Michigan Undergraduate Research Opportunity Program (UROP) for their support.

\section{References}

[1] Iijima S 1991 Nature 35456

[2] Lee S B, The A S, Teo K B K, Chhowalla M, Hasko D G, Milne W I, Amaratunga G A J and Ahmed H 2003 Nanotechnology 14192

[3] Buldum A and Lu J P 2003 Phys. Rev. Lett. 91236801

[4] Chhowalla M, Teo K B K, Ducati C, Rupesinghe N L, Amaratunga G A J, Ferrari A C, Roy D, Robertson J and Milne W I 2001 J. Appl. Phys. 905308

[5] Gao H, Wu X B, Li Ji T, Wu G T, Lin J Y, Wu K and Xu D S 2003 Appl. Phys. Lett. 833389 
[6] Kong J, Franklin N R, Zhou C, Chapline M G, Peng S, Cho K and Dai H 2000 Science 287622

[7] Farhat S, de la Chapelle M L, Loiseau A, Scott C D, Lefrant S, Journet C and Bernier P 2001 J. Chem. Phys. 1156752

[8] Waldorf E I, Waas A M, Friedmann P P and Keidar M 2004 J. Appl. Phys. 952749

[9] Bower C, Zhou O, Zhu W, Werder D J and Jin S 2000 Appl. Phys. Lett. 772767

[10] Wildoer J W G, Venema L C, Rinzler A G, Smalley R E and Dekker C 1998 Nature 3915962

[11] Gamaly E G and Ebbesen T W 1995 Phys. Rev. B 522083

[12] Iijima S, Ajayan P M and Ichihashi T 1992 Phys. Rev. Lett. 69 3100

[13] Saito Y, Yoshikawa T and Inagaki M 1993 Chem. Phys. Lett. 204277

[14] Colbert D T and Smalley R E 1995 Carbon 33921
[15] Zhou D and Chow L 2003 J. Appl. Phys. 939972

[16] Ando Y, Zhao X, Hirahara K, Suenaga K, Bandow S and Iijima S 2000 Chem. Phys. Lett. 323580

[17] Beilis I, Keidar M, Boxman R L, Goldsmith S, Heberlein J and Pfender E 1999 J. Appl. Phys. 86114

[18] Beilis I I, Keidar M, Boxman R L and Goldsmith S 2000 Phys. Plasmas 73068

[19] Nitter T 1996 Plasma Sources Sci. Technol. 593

[20] Keidar M and Beilis I I 1999 IEEE Trans. Plasma Sci. 27810

[21] Reimann K U 1991 J. Phys. D: Appl. Phys. 24493

[22] Keidar M, Beilis I, Boxman R L and Goldsmith S 1996 IEEE Trans. Plasma Sci. 24226

[23] Winske D and Jones M E 1995 IEEE Trans. Plasma Sci. 23 188

[24] Odom T W, Huang J L, Kim P and Lieber C M 1998 Nature 39162 